\section{Elementary Particle Physics - Where is it Going?}

\author{
Herwig Schopper, CERN, Geneva \\ Based on a talk given to the Council of the European Physical Society, 1 April 1982
}

The main objective of elementary particle physics is to investigate the structure and the origin of matter. During the past few centuries one has tried to understand the order which exists in Nature in terms of "ultimate" constituents and forces which act between these constituents.

Penetrating ever deeper into the microcosmos, one has identified smaller and smaller ultimate constituents. The indivisible "atoms" of the chemists of the last century were disintegrated by the physicists into nuclei surrounded by an electron cloud. The nuclei, it was found, are composed of protons and neutrons, and nowadays we believe that these particles are each made up of three quarks. There is, however, already speculation that even these particles could be composed of still smaller constituents. Hence we face the following logical difficulty: Either the divisibility of matter can be continued indefinitely and there are no ultimate constituents, or we find at last particles which have no internal structure and hence also no spatial extension and which correspond to mathematical points. But how can we understand that such mathematical points have a mass, an electric charge, spin etc. ?

As to the forces acting between these constituents, why are there several? (Indeed, we cannot be sure that we know them all.) What are their fundamental differences and relations, and might it be possible to unify them all into one fundamental force?

The most fundamental question which one would like to answer eventually, is why there are laws in Nature at all; why is Nature ordered and not simply in a state of chaos, and how is it possible from the laws of Nature, which are extracted from past experience, to predict events in the future?

It is my impression that in order to solve some of these problems or at least to get closer to a solution, it will be necessary to change radically our present concepts. But after all, all great break-throughs in physics were linked to a change in our way of thinking and it is precisely this sharpening of our mind, imposed on us by Nature, which always has had consequences far beyond the limits of physics.

\section{Ever Higher Energies}

Penetrating deeper and deeper into the microcosmos became possible only because higher and higher energies were available to elementary particle physicists. There are essentially three reasons why higher energies are necessary.

1. The resolution determining the smallest details which can be "seen" is proportional to the wave length of the "light" used, and since particles are used as probes, the wave length is given by de Broglie's relation $\lambda=h / p$. With the highest energies available today, details down to $10^{-16} \mathrm{~cm}$ can be resolved - a distance corresponding to $1 / 1000$ of the proton radius.

2. According to Einstein's relation $E=m c^{2}$ ( $E=$ energy, $m=$ rest mass of a particle, $c$ $=$ velocity of light) higher energies are necessary to produce heavier particles. Heavy particles have many decay channels into lighter particles and therefore usually are very short-lived. As a result they are not easily observed in Nature, and have to be produced artificially. To know about the existence of such particles and their properties is essential to understand the structure of matter. With presently available energies, particles up to about 20 proton masses can be produced which is not sufficient, as we shall see.

3. To understand different interactions it is necessary to study them not only at low energies (low frequencies), but also at high energies. Our understanding of electromagnetic interactions, for example, would be very fragmentary if we had explored experimentally only the electrostatic low-energy limit.

The only way to achieve the necessary high energies or, more explicitly, energy concentrations, is to accelerate particles by electromagnetic fields, and because the acceleration procedure takes of the order of seconds, the particles must be stable and charged. That leaves us with protons and electrons only and their respective anti-

Table 1. - The World's Principal Particle Accelerators

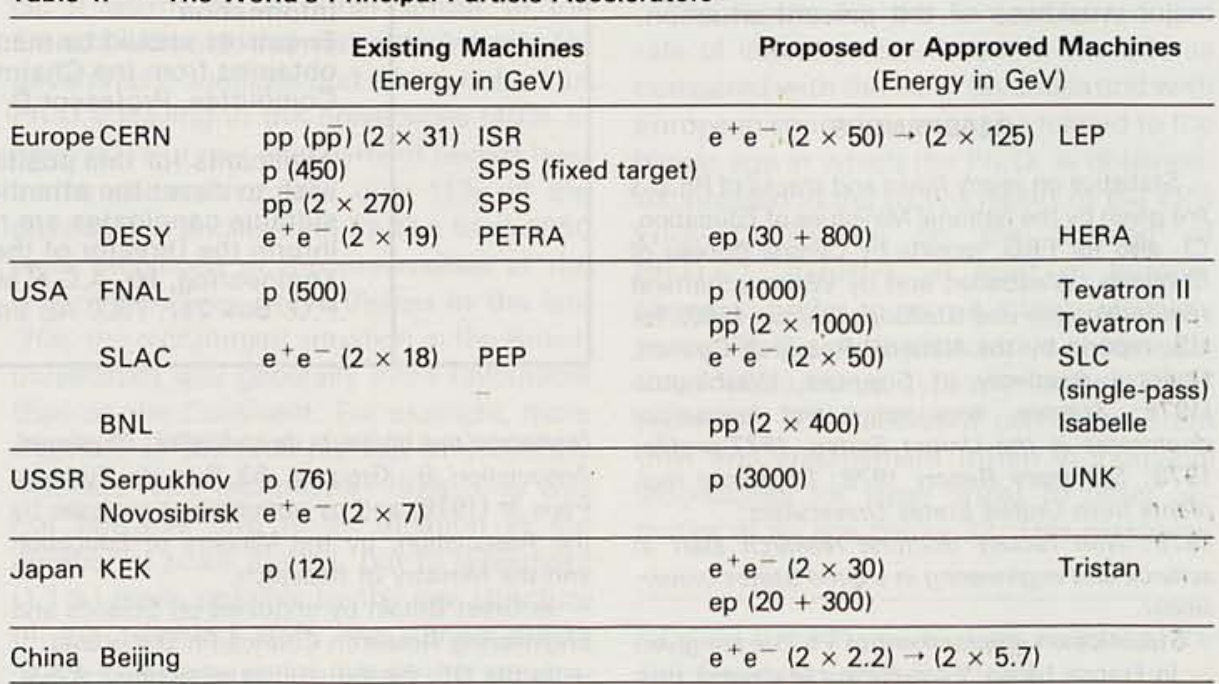

particles. As probes, protons and electrons behave in a complementary way.

Protons are relatively easy to accelerate and in a synchrotron the energy is limited by the product $B \times R(B=$ the magnetic field guiding them on a circle, $R=$ the radius of the orbit). Hence the momentum increases linearly with $B$ or $R$, and besides increasing the radius to achieve higher momenta, one tries to use superconducting technology to increase $B$. The disadvantage of the proton is its very complicated structure. From lepton scattering we know that the proton contains not only three "valence quarks", but, in addition, gluons which bind the quarks together and so-called "sea quarks" which are due to vacuum polarisation. As a consequence, collisions involving protons are rather difficult to interpret and extracting from them the fundamental information on collisions between the constituents of matter, e.g. quarks or gluons, is not easy.

This difficulty does not exist with electrons which, to our present knowledge, behave like mathematical points down to dimensions of $10^{-16} \mathrm{~cm}$. On the other hand, they are difficult to accelerate in a circular accelerator, as the centripetal acceleration leads to the emission of synchrotron radiation. The energy loss due to synchrotron radiation is proportional to $E^{4} / R$ (where $E$ $=$ energy of the electrons and $R=$ radius of their orbit): This energy loss must be compensated by accelerating fields and the $\mathrm{RF}$ power to produce these fields is proportional to $E^{s}$. To compensate this high power of $E$ by increasing the radius is not very efficient. Indeed, by doubling the radius the electron energy can be increased by only about $20 \%$. Therefore, it is not suprising that the energies imparted to electrons in circular accelerators are much lower than to protons (see Table 1) and the highest energies for electrons have been obtained in the 2-mile linear accelerator SLAC in Stanford where synchrotron radiation losses are avoided. However, it seems difficult to increase the energies of 
electrons by an order of magnitude in linear accelerators, and in particular it does not help in their accumulation and retention in storage rings. The only way out is to increase the radius by considerable factors, and this is the reason why the new electron-positron storage ring project at CERN, called LEP, will have a circumference of about $27 \mathrm{~km}$.

Positrons have been used in accelerators and storage rings for quite a number of years because they are comparatively easy to produce. Antiprotons, however, are difficult to produce because of their large mass and above all it requires special provisions to accumulate a sufficient number with sufficiently equal momenta to be used in an accelerator. This became possible for the first time last year at CERN and will be described below.

There are two ways of using accelerated particles:

a) In fixed target accelerators the accelerated protons or electrons are directed against nuclei (mostly hydrogen) at rest. The disadvantage is that according to relativistic kinematics, the available centre-ofmass energy increases only with the square root of the laboratory energy. On the other hand, in these collisions secondary particles are produced from which secondary beams can be derived, including electron beams with energies up to about $300 \mathrm{GeV}$. These cannot be obtained in any other way. Secondary beams allow many different processes to be investigated which could not be studied otherwise.

b) The other possibility is to make two particle beams collide head-on, in which case the centre-of-mass energy is just the sum of the energies of the two colliding beams. The disadvantage is that because of the small cross-sections, the chances of a collision are very small, and so far the most economic way is to store the beams in storage rings where they circulate for hours or even weeks increasing thereby the chances enormously.

If particles of the same kind are required to interact, two interleaved magnetic rings with opposite magnetic fields are necessary. The proton-proton collider, ISR at CERN, Fig. 1, is the first and so far the only project of this kind for protons. DORIS at Hamburg was originally built as a two-ring collider in order to observe electron-electron collisions, but it has never been used in this mode of operation.

More interesting and much simpler are storage rings where particles and antiparticles collide. Because of their opposite charge, only one magnetic ring is necessary. The two largest $\mathrm{e}^{+} \mathrm{e}^{-}$storage rings are PETRA in Hamburg (energy per beam $19 \mathrm{GeV}$, to be increased to about $22 \mathrm{GeV}$ ) and PEP at Stanford (beam energy 18 $\mathrm{GeV}$ ). Proton-antiproton collisions in storage rings were produced in 1980 for the first time at CERN, a remarkable achievement which I should like briefly to describe.

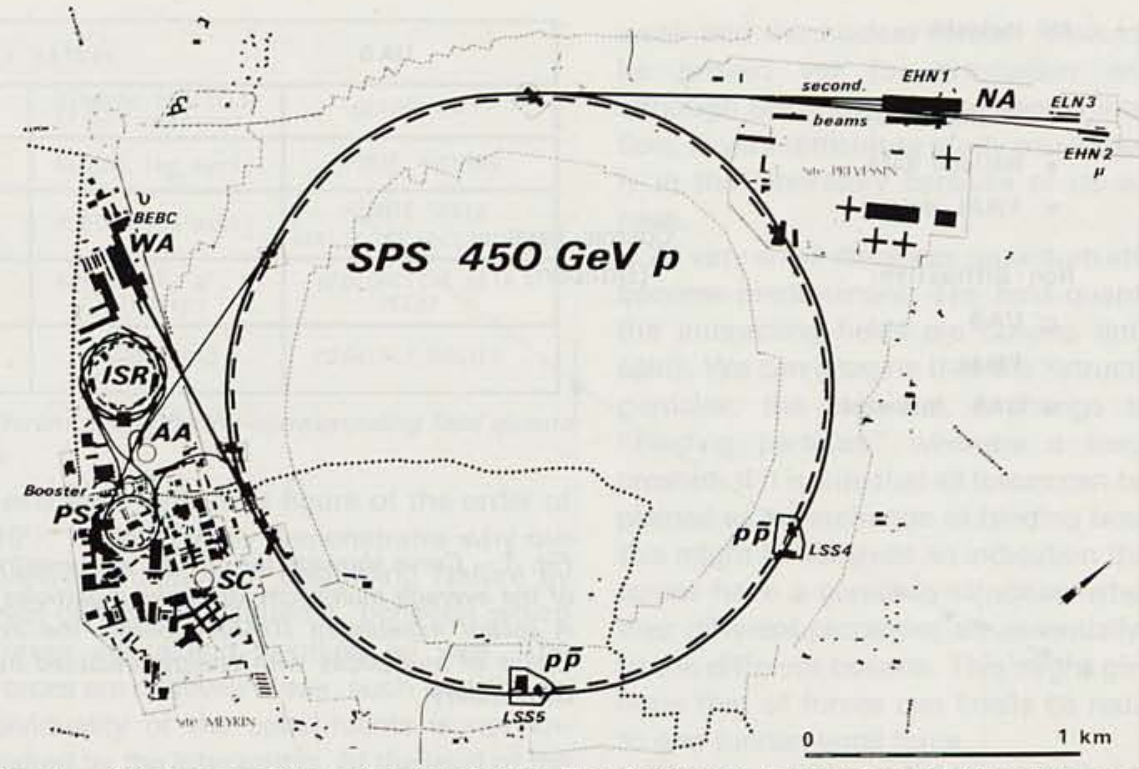

Fig. 1 - Site layout of the antiproton facility. The Antiproton Accumulator (AA) and the complex network of transfer tunnels were completed during the 1980 shutdown of the $450 \mathrm{GeV}$ Super Proton Synchrotron (SPS).

\section{CERN Proton-antiproton Project}

During the past few years, the SPS at CERN (Fig. 1) which for fixed target operation can accelerate protons up to $450 \mathrm{GeV}$, has been converted to a $\mathrm{p} \overline{\mathrm{p}}$ collider facility with a maximum energy of $270 \mathrm{GeV}$ per beam. For continuous colliding beam physics, the maximum energy is lower than for cycling fixed target operation because of the limitation of the power supplies. The centre-of-mass energy in proton-antiproton collisions which was achieved for the first time in 1981 is $2 \times 270=540 \mathrm{GeV}$, the highest energy ever produced in a laboratory. At the heart of this project is the system for producing "cooled" antiprotons.

Protons originally accelerated in the PS to $25.6 \mathrm{GeV}$ hit a metal target where among other particles, antiprotons with an average momentum of $3.5 \mathrm{GeV}$ are produced. The main difficulty is that these antiprotons are produced with momenta differing in direction and magnitude such that only a very small number could be injected - Reproduction of the centre of a $p \bar{p}$ interaction as recorded in a streamer chamber picture. (Experiment UA5, a Bonn/Brussels/Cambridge/CERN/Stockholm collaboration).

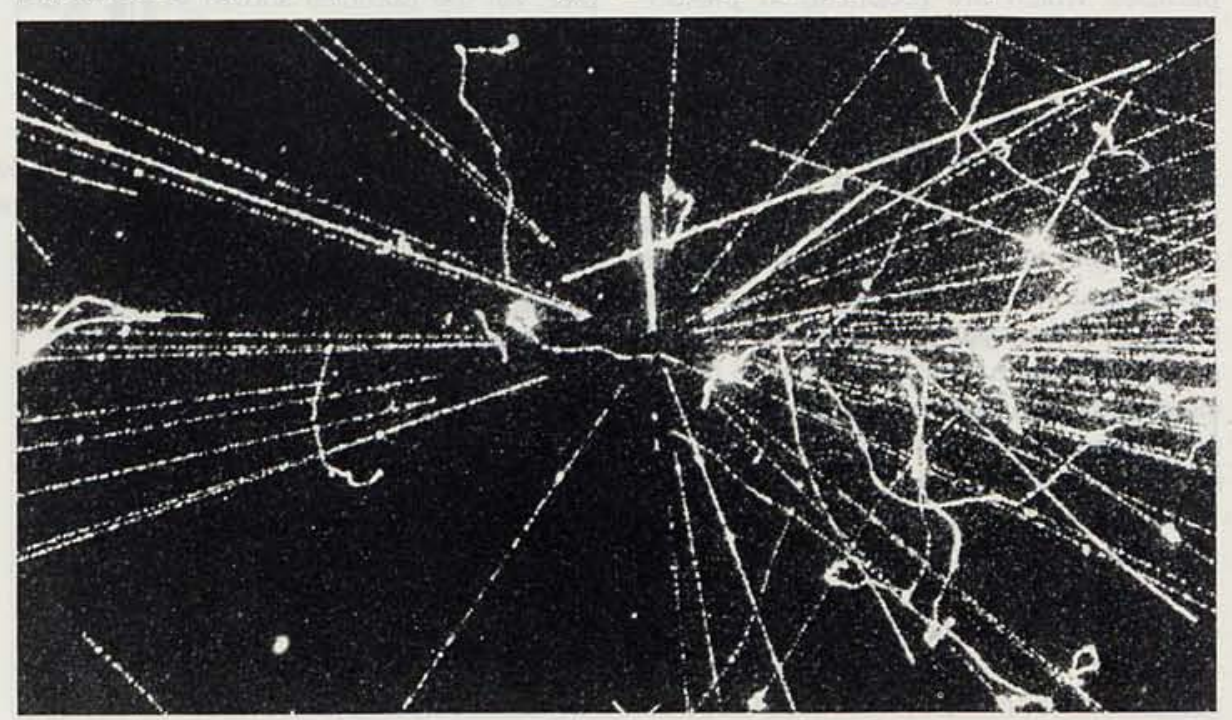

directly into an accelerator. To overcome this, a special ring, the Antiproton Accumulator Ring (AA) has been built whose task is to accumulate the antiprotons and $\mathrm{cool}$ them, which means homogenize their momenta. Seen from the average antiproton the other antiprotons have random velocities and their motion can be interpreted as a temperature. Homogenizing the momenta in direction and magnitude corresponds therefore to a cooling process. Cooling requires some interaction with the outside as Liouville's theorem states that phase space density cannot otherwise be reduced. But even with interference from the outside the cooling process seems a kind of Maxwellian demon.

In the ingenious system invented and developed at CERN which goes under the name of stochastic cooling, at a certain point of the circumference of the AA ring, a probe detects any deviation of a bunch of particles from the ideal orbit. A signal is passed across the diameter of the ring to a place on the opposite side and when the 
All inelastic:

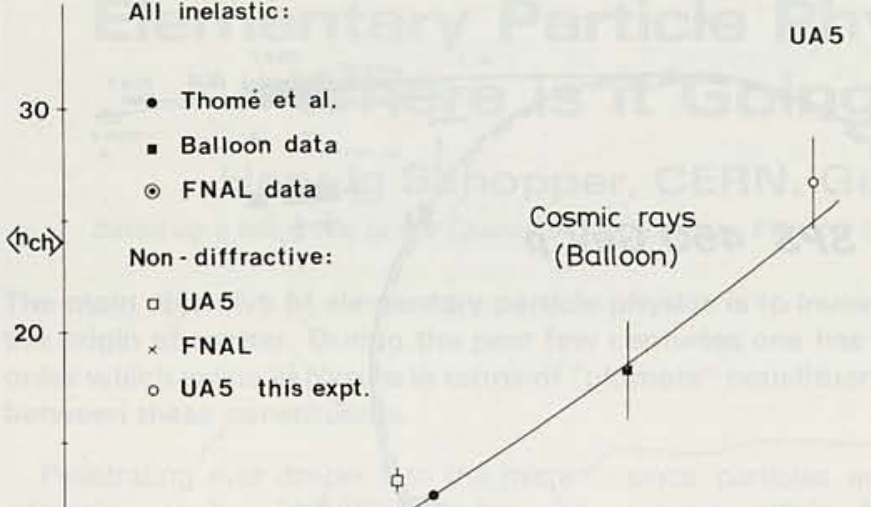

Fig. 3 - Curve showing the change with energy of the average multiplicity of charged particles. A recent experiment (UA5) confirms the increase of multiplicity with energy predicted by $Q C D$ theory.

$0+1$,

particle bunch arrives a kicker applies a correcting signal. In this way, cooling can be achieved in all six dimensions of phase space. The method works so effectively, it takes only a few seconds to produce a homogenous proton beam. In reality the process is much more complicated, and indeed would not work for an infinite number of particles; fluctuations of a finite number of particles are necessary, hence the name stochastic cooling.

The antiprotons are taken through a beam transfer loop back to the PS (Fig. 1) where they are injected in the opposite sense, accelerated up to $26 \mathrm{GeV}$, and then taken through a transfer channel to the SPS. To get a sufficient number of antiprotons takes about a day, which implies that the whole system with its many injections and transfer systems has to work extremely reliably. The first proton-antiproton collisions were observed in August 1981, and the experimental programme started in the autumn of the same year with, in total, five collaborations to do experiments.

One of the remarkable features of these very high energies is the large number of particles which are produced in protonantiproton collisions (see Fig. 2). One can really speak of the creation of matter and we might be very close to the conditions under which matter was produced during the big bang at the origin of the Universe. In Fig. 3 the average number of particles produced in proton-antiproton collisions is shown as a function of the centre-of-mass energy. In the coming years the SPS will provide a unique instrument for the physicists, and it can be hoped that some fundamental questions which I shall mention later might be clarified.

\section{Next Generation of Accelerators}

In Table 1, the new projects which are under construction or have been approved are listed. A remarkable tendency can be seen. Whereas in the past a certain duplicaticles a consts, meaning

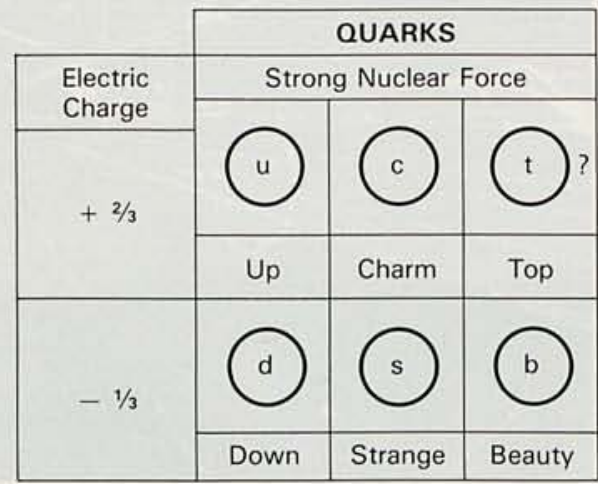

Table 2. - Binding Energy of Composite Units

\begin{tabular}{|c|c|c|}
\hline $\begin{array}{l}\text { Composite } \\
\text { Unit }\end{array}$ & Constituents & $\begin{array}{c}\text { Binding Energy } / c^{2} \\
\text { Rest Mass } \\
\text { of Constituents }\end{array}$ \\
\hline Molecule & Atoms & $10^{-10}$ \\
\hline $\begin{array}{l}\text { Atomic } \\
\text { Nucleus }\end{array}$ & $\begin{array}{l}\text { Hadrons } \\
(p, n, \wedge)\end{array}$ & $10^{-2}$ \\
\hline Hadron & Quarks & $\approx 1$ \\
\hline Quark & $\begin{array}{l}\text { Rishons, } \\
\text { Haplons }\end{array}$ & $\gg 1$ \\
\hline
\end{tabular}

that they cannot be produced as single particles but only in particle-antiparticle pairs. There are two classes of particle: the leptons which do not feel the nuclear force and the quarks which do. Another major difference is that quarks have $1 / 3$ charge whereas leptons have integer electrical charges. The particles in the first line of the scheme differ from those in the second line by one unit of electric charge. The two particles in each column form a family with respect to weak interactions in the sense that they can be transformed into each other. Thus, in weak processes a u quark can be transformed into a $\mathrm{d}$ quark and vice versa or an electron into an electron neutrino, etc. The mass of the leptons increases from left to right. Thus, besides the electron, a heavy electron (which is usually called a muon) is known and a couple of years ago a super-heavy electron, the $\tau$ particle, was detected. Each of these electron-like particles has its own neutrino. Although experimental upper limits on the masses of the neutrinos are small, one of the most interesting questions is whether the mass of these neutrinos is exactly zero or not.

Originally, three quarks ( $u, d$ and s) were identified, but in the seventies the charm quark and the beauty quark were discovered. Because of the supposed symmetry between leptons and quarks most physicists are convinced that a sixth quark, the top quark, must exist. As we have so far no understanding of the rules governing the masses of these particles, it is not possible to predict the mass of the top quark. For a certain time it was hoped that it could be produced with PETRA but it seems to be heavier than the available energy. Maybe it can be found with the $\mathrm{p} \overline{\mathrm{p}}$ collider or LEP.

Fig. 4 - The 'periodic system' of elementary particles showing the two families, the quarks and the leptons, which are thought to be the fundamental constituents of matter.

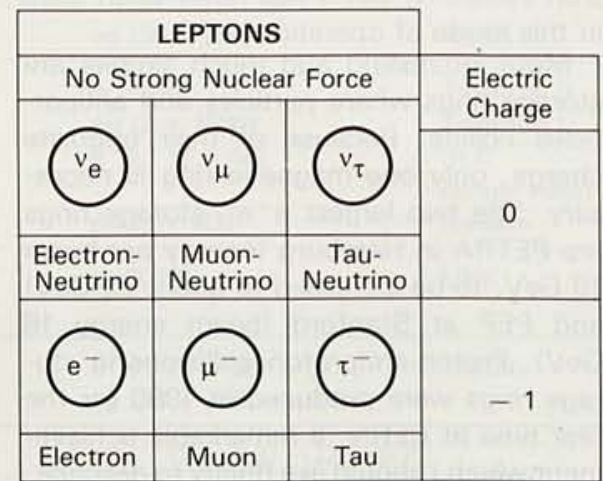




\begin{tabular}{|c|c|c|c|}
\hline \multicolumn{4}{|c|}{ THE FORCES IN NATURE } \\
\hline TYPE & $\begin{array}{l}\text { INTENSIIY OF FORCES } \\
\text { (Decreas ing order) }\end{array}$ & $\begin{array}{l}\text { BINDING PARIICLE } \\
\text { (Field quantum) }\end{array}$ & OCCURS IN \\
\hline STRONG NUCLEAR FORCE & 21 & GLUONS (no mass) & ATOMIC NUCLEUS \\
\hline ELECTROMAGNETIC FORCE & $\therefore \frac{1}{1000}$ & PHOTON (no mass) & $\begin{array}{c}\text { ATOHIC SHELL } \\
\text { APPLIC. OF ELECTRICITY }\end{array}$ \\
\hline WEAK NUCLEAR FORCE & $\frac{1}{100000}$ & $\begin{array}{l}\text { BOSONS } Z^{0}, \mathrm{H}^{+}, \mathrm{W}^{-} \\
\text {(heavy) }\end{array}$ & $\begin{array}{l}\text { RADIOACTIVE BETA } \\
\text { DECAY }\end{array}$ \\
\hline GRAVITATION & $=10^{-30}$ & GRAVITON? & HEAVENLY BODIES \\
\hline
\end{tabular}

Fig. 5 - The four forces in nature. Besides their different strengths the corresponding field quanta determine the different properties of the interactions.

Looking at the scheme of Fig. 4, a number of fundamental questions immediately arise: why are there six leptons and six quarks? Why is six a fundamental number not four or eight? Although no heavier lepton than the $\tau$ has been found so far (the energies are not sufficient to produce heavier quarks than the b) it cannot yet be excluded that there is an infinite number of leptons and quarks. Such a series of particles could be interpreted as excited states of one fundamental system. On the other hand, symmetry considerations could speak in favour of a finite number of leptons and quarks, but then we must ask the question: why are there two classes of particle? Several theorists have speculated that quarks and leptons might not be the ultimate constituents, but that there might be a deeper layer of matter. They have postulated even smaller particles (sometimes called rishons or haplons) out of which both quarks and leptons can be composed. Such a composition of quarks and leptons would imply that the latter particles have an internal structure and above all a finite radius.

So far, experiments at PETRA have shown that both leptons and quarks behave like point-like particles down to dimensions of about $10^{-16} \mathrm{~cm}$. This does not yet exclude completely an internal structure of leptons and quarks, and only experiments at higher energies will be able to evaluate all these speculations. However, independently of whether some constituents of quarks and leptons can be found, I believe that we have come to a principal limit in subdividing into smaller and smaller parts, the constituents of matter. If this should turn out to be true, it might be the most fundamental discovery of elementary particle physics in this century.

There are two indications for such a limit. First, it has not been possible to isolate individual quarks, although as I shall explain below, this might be due to the properties of the nuclear forces. Second, there seems to be an even more general argument. If we consider the ratio between the binding energy and the rest masses of the constituents of certain composite structures (see Table 2) we find that this ratio increases as the constituents become smaller, and it has to be much larger than unity for sub-constituents of quarks and leptons (estimates indicate a figure of the order of $10^{6}$ ). Table 2 clearly demonstrates why our previous method of describing Nature by forces are acting, worked so well. The dividuality of the constituents is not impaired by the interaction. At the level of the quarks this situation changes dramatically. For sub-constituents of quarks the interaction between these particles has become so strong that it seems difficult to continue to consider these constituents as individual particles. The philosophical consequence, of course, would be that a further subdivision of matter into smaller particles is still conceivable, but loses practically its meaning. We might be forced to abandon our method of describing Nature by individual particles and their interactions.

\section{Forces at the Origin of Matter}

All this indicates that an understanding of the forces which we find in Nature might be the clue to an understanding of the structure of matter. The forces which we know today are shown in Fig. 5 where they are ordered according to their strength. We believe that the forces do not act directly over long distances, but that they are mediated by fields which represent a special state of the space-time continuum. This was first demonstrated for the electromagnetic interactions, but we have evidence nowadays that it is also true for the well-defined constituents, between which forces are relatively weak, such that the in- weak and the nuclear forces. It could not be proved yet for gravitation which, although being the longest known interaction, is very difficult to study experimentally in the laboratory because of its weakness.

At very small distances, quantum effects become predominant. The field quanta of the interacting fields are bosons (integer spin). We can imagine that the "structure" $p$-rticles, the fermions, exchange these "binding particles" whereby a force is created. If it is true that all forces can be explained as an exchange of binding bosons, this might be taken as an indication that all forces have a common structure whereas their different properties are essentially due to the different bosons. This might give us hope that all forces can finally be reduced to one fundamental force.

The best known field quantum is the photon which has no mass and spin 1. Three years ago the binding particle of the strong interaction, which has the name gluon, was detected at PETRA. Like the photon, it has no mass and spin 1. Both carry no electric charge.

For the weak interaction, the binding particles, the intermediate bosons, must have completely different properties. In weak processes, like nuclear beta decay, the charge of the interacting particles changes (e.g., a neutron is converted into a photon) so that the intermediate bosons $\mathrm{W}^{+}$and $\mathrm{W}^{-}$must have an electric charge. As the range of this interaction is extremely short, the mass of these particles must be very high according to the uncertainty principle, and it has not been possible to detect them experimentally so far.

A major step was achieved when Glashow, Salam and Weinberg developed a theory which tried to unify the electromagnetic and the weak interaction. One of the predictions of this theory was that besides the two charged intermediate bosons, there should also be a neutral boson $Z^{\circ}$ which in a way is nothing other

\section{(5) \\ Physics Institute of the University Zürich}

The experimental group of the Physics Institute of the University Zürich working in collaboration with the ETH-Zürich at the Swiss Institute for Nuclear Research (SIN) is looking for a

\section{PHYSICIST OR PHYSICS ENGINEER}

The group is building a spectrometer for rare and forbidden $\mu$ - and $\pi$ decays. Candidates with experience in particle detectors especially wire chambers are preferred.

The position becomes available from January 1983 for three years with possible extensions. Salary will depend upon qualifications and experience.

Please reply, stating all pertinent information and naming references, to: Prof. Roland Engfer

Physik-Institut, Universität Zürich

Schönberggasse 9

CH-8001 Zürich, Switzerland 
than a heavy proton. In 1973 , the existence of the so-called neutral currents were established at CERN which are an indirect indication for the existence of $Z^{\circ}$. Many experiments at relatively low energies are in full agreement with this theory; however, what is necessary is its verification at high energies. The detection of intermediate bosons whose mass is predicted to be 80-90 times the proton mass would be an important step in this direction. The only accelerator which has enough energy is the proton-antiproton collider at CERN and therefore a discovery of these particles in the coming years might be possible. A detailed study of their properties and a full test of the unifying theory will, however, only be possible when LEP comes into operation.

\section{New Kind of Charge: Colour}

The next question we might ask is whether the nuclear force could also be integrated in this unification process. Indeed, there are some indications. However, before such a step can be made it seems necessary to understand better the strong force itself. For many decades only phenomenological descriptions of the nuclear force were available and it is only during the past few years that a theory has been developed for the nuclear force analogous to quantum electrodynamics (QED). The theory is called quantum chromodynamics (QCD). Recent experiments support this theory, although many details and also some fundamental questions remain to be clarified.

The name of QCD has the following origin. In contrast to the electrical charge it is supposed that for the strong interactions there are three different charges and each charge has its own opposite, or anticharge (see Fig. 6). A neutral state can be produced as in the electric case by a combination of the charge and its proper anticharge. In QCD however, a new possibility exists. If three charges are combined (without involving an anticharge) one can also produce a neutral state. This corresponds to the known procedure of mixing colours where three basic colours can give a white colour. Because of this analogy the three charges of the strong force are designated by colours, e.g. red, green or blue with their anticolours antired, antigreen, antiblue. Carriers of these anticharges and hence sources of the strong force are the quarks, whereas leptons do not carry any colour charge.

So far, one has found in Nature only neutral, white states. This implies that only two kinds of quark "molecules", which are called hadrons, can be composed. Either a quark and an antiquark are bound together which is called a meson, or the other possibility is that three quarks form a particle (called baryons) with the proton and the neutron as the best known examples. No other combinations have been found so

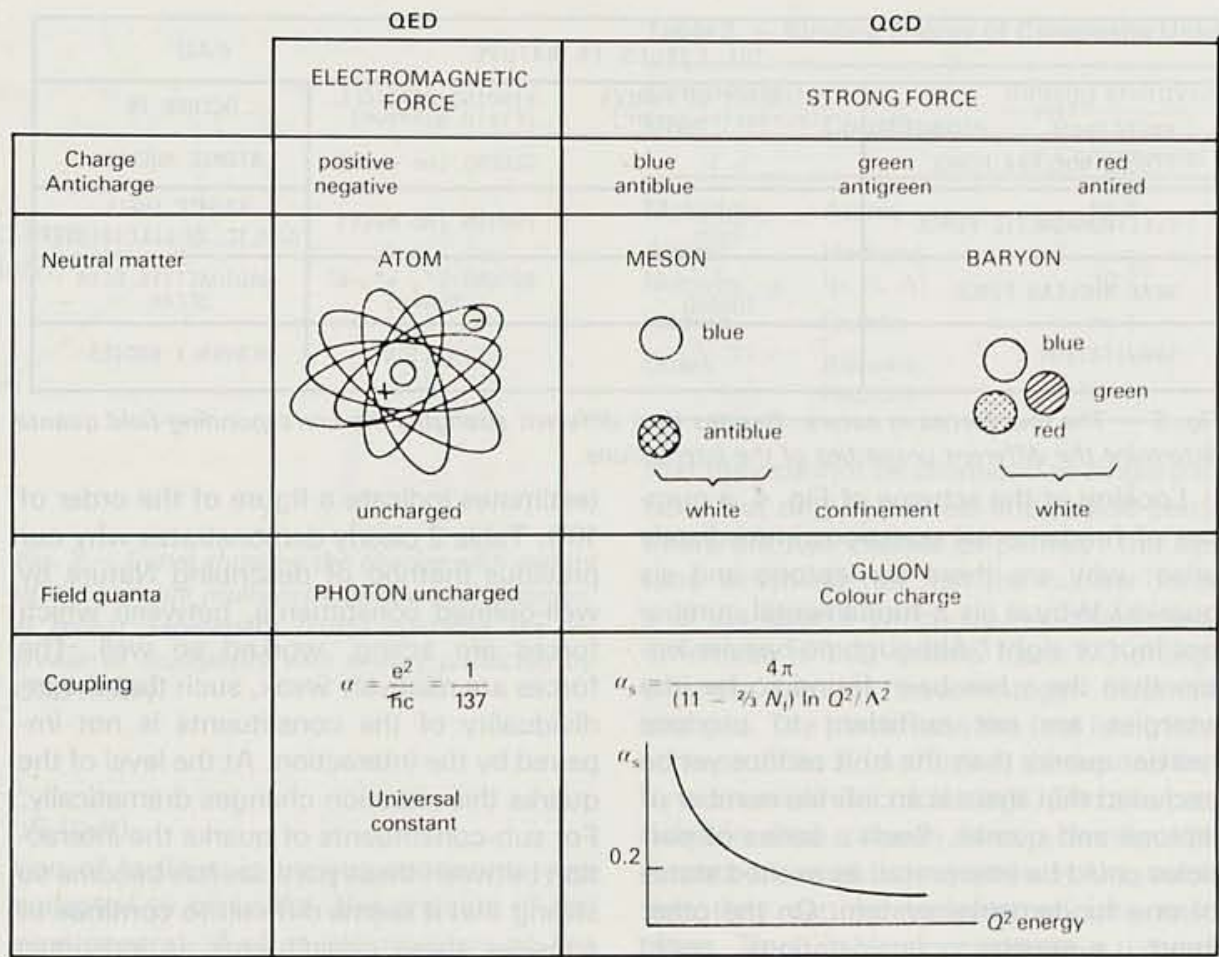

Fig. 6 - A comparison between Quantum Electrodynamics (QED) and Quantum Chromodynamics $(Q C D)$, the Gauge field theories governing, respectively, the electromagnetic force and the strong force. In $Q C D$ there are three charges (and their anticharges) and consequently there are two different ways to form a neutral ('white') state. The coupling $\alpha$ in QED is a universal constant whereas $\alpha_{s}$ changes with energy $Q . \Lambda$ is the fundamental constant characterizing $Q C D$.

far, implying that the quark "chemistry" is very simple. As mentioned above, the field quanta of the strong force are called gluons, and one essential difference between QED and QCD is that the photons do not carry an electric charge whereas the gluons are colour charged. This implies that a direct gluon-gluon interaction and even bound gluon states are possible. This important prediction of QCD has not been definitely confirmed experimentally yet, although there exist some indications for bound gluon states (gluon balls).

A very remarkable experimental fact is that so far no free colour charges have been produced, and that no free quarks and no free gluons have been observed. The question now is if our available energies are just not high enough to break the colour binding or if there is a fundamental principle which forbids free colour states and allows only the existence of neutral states in Nature.

The answer to these questions might be linked to another fundamental difference between QED and QCD (Fig. 6). The strength of the coupling between an electrical charge and the electro-magnetic field is determined by the size of the elementary charge of the dimensionless quantity derived from it $\alpha=e^{2} / \hbar c$. This fine structure constant is a universal quantity and is in particular independent of the interaction energy. In $Q C D$, it can be derived from very general principles that the corresponding coupling constant $\alpha_{\mathrm{s}}$ is indeed not a constant but depends on the interaction energy in a logarithmic way. At large energies it is small, but increases with decreasing ener- gy. The energy dependence is characterized by a parameter $\Lambda$ (see Fig. 6) and this parameter is the characteristic constant describing the strong interactions and not $\alpha$ itself. An experimental determination of $\Lambda$ is difficult as the logarithmic dependence on energy is rather weak. Last year several experiments at CERN could determine this quantity and it was found that the order of magnitude is about $\Lambda \cong 100 \mathrm{MeV}$ corresponding to a length of about $3 \mathrm{fm}$.

The change of the coupling with interaction energy has a very important consequence. If quarks interact at small distances, the interaction energy is high, the coupling is low, and the quarks behave almost like free particles lasymptotic freedom). In this case, QCD behaves almost like QED and indeed many formulae of QED can simply be adapted to QCD. The situation is completely different, however, for large distances between the quarks. Then the coupling increases and if one tries to separate two quarks, quark-antiquark pairs are created, which combine with the original quark to colourless, white states. This is analogous to breaking a magnet where at the breaking point a new pair of North and South poles is formed, such that the two pieces of the magnet are both dipoles and no monopoles could be produced. If this "confinement" of quarks is corroborated experimentally and could be better understood theoretically, it might be the most important discovery in elementary particle physics. Apart from its consequences for a better understanding of the strong interactions it would imply that we have come to a principal limit of sub- 
dividing the constituents of matter into smaller and smaller parts.

\section{From Democritus to Plato}

I should like to come back to the questions why there are four interactions in Nature and what their relation might be. One big break-through in physics during the past century was the recognition that electric and magnetic phenomena are just two different aspects of one underlying unified force. Can we hope to unify eventually all four forces?

As has been mentioned above, it seems that all forces can be explained by the same principal mechanism, that is they are mediated by the exchange of intermediate bosons between the constituents of matter which are fermions. One difficulty in unifying forces might be their strength. How can it be that forces with such different coupling strengths can be the offspring of the same basic phenomenon? It seems that this difficulty can be overcome in the following way. The coupling strengths indicated in Fig. 6 are those experimentally determined at low energies. However, it turns out that the strength of the weak interaction increases with interaction energy and at energies corresponding to the masses of the $\mathrm{W}$ and $\mathrm{Z}$ its strength becomes comparable to the electro-magnetic interaction. Hence, at such energies corresponding to distances of about $10^{-17} \mathrm{~cm}$, the unification of these two interactions becomes possible.

On the other hand, we have seen that the strength of the strong interaction decreases with increasing energy and at energies of about $10^{15} \mathrm{GeV}$ it becomes comparable to the strength of the electroweak interaction. Hence at distances of about $10^{-29} \mathrm{~cm}$ a "grand unification" might become possible. We know very little about the quantum behaviour of gravitation, but some speculations indicate that this interaction could also be included in the unification at energies of about $10^{20}$ $\mathrm{GeV}$ or distances of about $10^{-34} \mathrm{~cm}$. This is certainly very far away from present-day experiments.

Is the endeavour to unify all known interactions in one fundamental force just a romantic dream of the physicists or has it a more general relevance? First, I should like to come back to the question of whether Nature can be described by independent, individual constituents and forces acting between them. Above, I gave some arguments why we might have to replace this concept by more general ones. A concept that seems to be more fundamental although being also more abstract are the Gauge fields. Indeed, it turns out that at the basis of all the mentioned theories describing the various interactions are Gauge fields. The practically infinite number of mathematically possible Gauge fields is restricted by the symmetry properties of these fields. It seems therefore that the first principles of describing and understanding Nature may not be little undestroyable bricks of matter, but rather abstract concepts, symmetries. It seems that modern elementary particle physics is taking us from Democritus to Plato which eventually might have important repercussions beyond the limits of physics.

If all forces can indeed be described by Gauge field theories it might in the end be possible to derive everything from just one fundamental Gauge field. This could have a far reaching consequence for our understanding of Nature. Each abstract field is related to a special structure of the spacetime continuum. It is difficult to conceive that several such structures should co- exist. Conceptually it certainly is much simpler if all interactions could be derived from just one space-time structure. Considering the interactions, one can furthermore find that the existence of matter as we know it, depends very critically on the detailed properties of all interactions. Changes in the coupling of one force or the elimination of one interaction would entail a collapse of matter. It is difficult to understand that this exact coordination of the interactions should be an accident and should not have a deeper connection. If this were so, a unified field theory could also help to solve the old problem of Hume, which was the question: why there are laws of Nature at all.

\begin{tabular}{|c|c|c|}
\hline 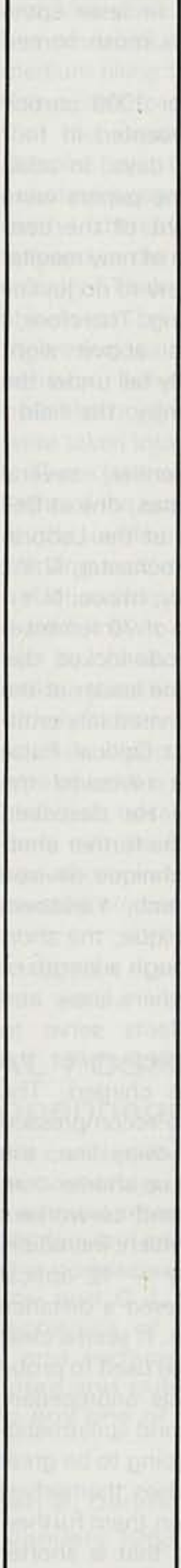 & 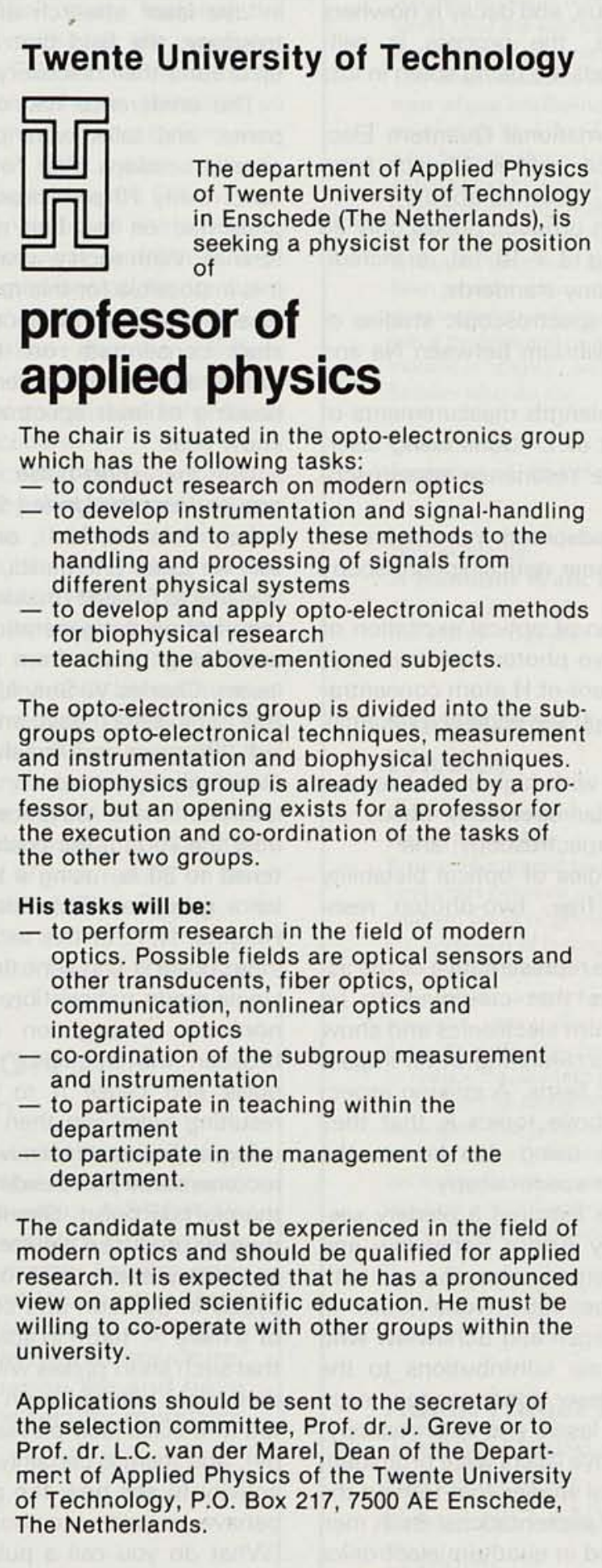 & • \\
\hline
\end{tabular}

